\title{
Uso della bioimpedenza come predizione di sopravvivenza nei pazienti in emodialisi
}

\author{
V. Terracciano, B. Di Iorio
}

\author{
Unità Operativa di Nefrologia e Dialisi \\ Azienda Sanitaria USL $n^{\circ} 3$ del Lagonegrese \\ PP.OO. di Lauria e Maratea $(\mathrm{Pz})$
}

analisi della bioimpedenza

(BIA) è una metodica strumentale che si è diffusa rapidamente nei Reparti di Nefrologia e Dialisi, poiché consente, al letto del paziente, in maniera rapida, semplice, riproducibile e poco costosa, di ottenere importanti dati sulla composizione corporea (1-4).

Pertanto, mediante BIA, è possibile stimare l'acqua corporea totale (TBW), utile supporto in emodialisi (HD) per una più precisa individuazione del "peso secco" e, fatto di crescente importanza proprio in HD, valutare lo stato nutrizionale del paziente.

Il presupposto fisico su cui si basa BIA è il seguente: l'applicazione di una corrente elettrica alternata, costante e di basso livello, ad un organismo vivente incontra una Impedenza (Z), ossia un ostacolo al passaggio della corrente stessa, che dipende sia dalla frequenza della corrente che dalle caratteristiche, specie di conducibilità elettrica, dell'organismo (5).

I tessuti magri, ricchi di acqua ed elettroliti, conducono facilmente la corrente, mentre il tessuto grasso è un cattivo conduttore (5).

$\mathrm{Z}$ consta di due diverse componenti: $\mathrm{Re}$ sistenza (Re) e Reattanza (Xc), misurate in ohms (5).

Re è la diretta espressione della quantità di acqua corporea, Xc della distribuzione tra spazio intra ed extra-cellulare e, quindi, è una misura indiretta delle membrane cellulari integre (5).

La BIA consiste, in pratica, nella misurazione di questi due parametri. L'esame, della durata di pochi secondi, viene effettuato con il soggetto sdraiato e con gli arti in abduzione. Le due coppie di elettrodi, necessarie per la misurazione, vengono applicate, rispettivamente, sul dorso della mano e del piede omolaterale (negli emodializzati, controlaterali al lato dell'accesso vascolare), mantenendo una distanza non inferiore a $5 \mathrm{~cm}$ tra l'elettrodo iniettore della corrente (distale) e quello sensore della caduta di voltaggio (prossimale).

Per la stima dei volumi corporei, i valori misurati di Re e Xc vengono elaborati da un software mediante equazioni matematiche, validate in una popolazione normale di riferimento. In particolare, Re è usata per misurare TBW (è stato calcolato un coefficiente di correlazione $>0.97$ con la metodica di diluizione isotopica) $(6,7)$. Dal valore di TBW viene derivato quello della massa magra (FFM), assumendo che circa il $73 \%$ dei liquidi è distribuito nei tessuti magri. Di conseguen$\mathrm{za}$, viene indirettamente determinata anche la massa grassa (FM), calcolata dalla differenza: peso corporeo - FFM.

$\mathrm{Xc}$ è proporzionale alla massa cellulare corporea (BCM): dal rapporto $\mathrm{Xc} / \mathrm{Rz}$ de- riva un $3^{\circ}$ parametro, l'Angolo di Fase (PA), misurato in gradi, usato nelle equazioni derivative per predire proprio BCM (8).

Ma la validità di tali equazioni in situazioni patologiche suscita notevole perplessità, dal momento che esse sono fortemente condizionate dallo stato di idratazione (9). Di qui la costante ricerca di indici, che potessero caratterizzare uno stato di "normalità bioelettrica", riferimento necessario per confrontare popolazioni diverse per patologia (10). Piccoli et al (11-14), utilizzando le misure elettriche $(\mathrm{Re}$ e $\mathrm{Xc})$, corrette per l'altez$\mathrm{za}$, hanno elaborato un metodo grafico (RXc graph) utile per la valutazione dello stato di idratazione (con il calcolo dei limiti di tolleranza bivariati al 50, 75 e $95 \%$, espressi graficamente come ellissi) specifico per sesso, in una popolazione di ben 726 adulti sani con range di età tra 15 e 85 anni.

Inoltre, gli stessi Autori, hanno valutato pazienti con insufficienza renale cronica (IRC), pazienti con sindrome nefrosica, pazienti obesi prima e dopo terapia dietetica, atleti, pazienti con colera prima e dopo correzione della disidratazione. Piccoli, ancora, è stato il promotore di uno studio multicentrico (Italian Cooperative HD-BIA Study Group) per la valutazione dello stato di idratazione in pre e in post HD (con l'uso di RXc graph) 
dei pazienti italiani (dati in corso di pubblicazione).

Talluri ha proposto un nomogramma (rappresentato dal confronto dei dati di Xc e quelli di PA) di normalità, derivato dall'analisi di 888 studenti universitari e 491 soggetti pediatrici (15).

Infine, è stato dimostrato che le misure elettriche differiscono nella popolazione normale sia in funzione del sesso che dell'età (16).

In età pediatrica, poi, non vi sono sufficienti dati di controllo nella popolazione normale. In Italia, a nostra conoscenza, a parte Talluri, solo il gruppo di De Palo ha valutato le misure elettriche in una popolazione normale pediatrica $(17,18)$. Da questa analisi traspare l'esigenza di poter dotarsi di indici di normalità delle misure elettriche sicuri ed efficaci.

A tal proposito, anche noi, in collaborazione con altri Gruppi, stiamo raccogliendo i dati (Re, Xc e PA) di soggetti normali, nel tentativo di elaborare veri e propri "Percentili" di normalità dei parametri elettrici, divisi per sesso e per fasce d'età. (Gior Ital Nefrol 1996; 13: 269).

\section{Bioimpedenza e mortalità}

Negli ultimi tempi è stata posta in luce, da vari Autori, la possibilità di usare i parametri bioelettrici, specie PA, come markers dello stato nutrizionale in HD e in Dialisi peritoneale (19-23).

In particolare, Chertow et al (20), p.e., hanno evidenziato un'alta correlazione
TABELLA I - DATI BIOELETTRICI, NUTRIZIONALI ED EFFICIENZA DIALITICA IN PAZIENTI MASCHI EMODIALIZZATI (MEDIA \pm DS)

\begin{tabular}{lccc}
\hline & Vivi & Morti & $p$ \\
\hline Età anagrafica (anni) & $55.17 \pm 11.51$ & $70.25 \pm 2.71$ & 0.001 \\
Età dialitica (mesi) & $87.50 \pm 58.42$ & $47.5 \pm 19.85$ & 0.001 \\
Albumina (g/dl) & $3.99 \pm 0.38$ & $3.63 \pm 0.75$ & $\mathrm{NS}$ \\
$\mathrm{nPCR}$ & $1.15 \pm 0.27$ & $1.11 \pm 0.28$ & $\mathrm{NS}$ \\
Kt/N & $1.39 \pm 0.27$ & $1.44 \pm 0.24$ & $\mathrm{NS}$ \\
Re-pre (ohms) & $458 \pm 79$ & $481 \pm 61$ & $\mathrm{NS}$ \\
Xc-pre (ohms) & $35 \pm 12$ & $28 \pm 11$ & $\mathrm{NS}$ \\
Re-post & $542 \pm 97$ & $556 \pm 52$ & $\mathrm{NS}$ \\
Xc-post & $50 \pm 18$ & $35 \pm 14$ & 0.05 \\
PA-post & $5.16 \pm 1.65$ & $3.6 \pm 1.03$ & 0.05 \\
Capacità & $551 \pm 215$ & $100 \pm 15$ & 0.001 \\
AEI & $10.11 \pm 3.31$ & $8.15 \pm 2.4$ & 0.001 \\
BMI-c & $5.07 \pm 2.22$ & 0.001 \\
\hline
\end{tabular}

TABELLA II - DATI BIOELETTRICI, NUTRIZIONALI ED EFFICIENZA DIALITICA IN PAZIENTI FEMMINE EMODIALIZZATE (MEDIA \pm DS)

\begin{tabular}{lccc}
\hline & Vivi & Morti & $p$ \\
\hline Età anagrafica (anni) & $58.37 \pm 15.91$ & $67.26 \pm 7.33$ & 0.05 \\
Età dialitica (mesi) & $67.23 \pm 50.72$ & $87.6 \pm 74.38$ & 0.05 \\
Albumina (g/dl) & $4.06 \pm 0.59$ & $3.8 \pm 0.56$ & 0.05 \\
nPCR & $1.19 \pm 0.26$ & $0.93 \pm 0.11$ & 0.05 \\
Kt/V & $1.53 \pm 0.36$ & $1.35 \pm 0.27$ & NS \\
Re-pre (ohms) & $551 \pm 106$ & $526 \pm 149$ & $\mathrm{NS}$ \\
Xc-pre (ohms) & $38 \pm 17$ & $24 \pm 7$ & 0.05 \\
Re- post & $650 \pm 125$ & $615 \pm 170$ & $\mathrm{NS}$ \\
Xc-post & $52 \pm 23$ & $29 \pm 8$ & 0.001 \\
PA-post & $7.77 \pm 3.02$ & $2.8 \pm 0.87$ & 0.001 \\
Capacità & $387 \pm 143$ & $274 \pm 145$ & $\mathrm{NS}$ \\
AEI & $9.18 \pm 4.01$ & $5.88 \pm 1.48$ & 0.001 \\
BMI-c & $6.88 \pm 4.15$ & $9.85 \pm 3.4$ & 0.001 \\
\hline
\end{tabular}

TABELLA III - DATI BIOELETTRICI E BIOIMPEDENZIOMETRICI-DERIVATI IN PAZIENTI ANZIANI CON IRC

\begin{tabular}{|c|c|c|c|c|c|c|}
\hline & & Maschi & & & Femmine & \\
\hline & $\operatorname{IRC}(n=12)$ & $\mathrm{HD}(\mathrm{n}=6)$ & Morti $(n=3)$ & $\operatorname{IRC}(n=12)$ & $\mathrm{HD}(n=6)$ & Morti $(n=1)$ \\
\hline $\operatorname{Re}$ & $483 \pm 111$ & $426 \pm 62$ & $581 \pm 24$ & $555 \pm 78$ & $508 \pm 52$ & $510 \pm 5$ \\
\hline $\mathrm{Re} / \mathrm{H}$ & $296 \pm 60$ & $255 \pm 41$ & $347 \pm 7$ & $362 \pm 55$ & $326 \pm 35$ & $345 \pm 5$ \\
\hline $\mathrm{Xc}$ & $39 \pm 8$ & $30 \pm 5$ & $36 \pm 13$ & $43 \pm 12$ & $35.5 \pm 8.8$ & $31.0 \pm 4 * * *$ \\
\hline $\mathrm{Xc} / \mathrm{H}$ & $24 \pm 5$ & $17 \pm 3^{*}$ & $16.02 \pm 5.2^{*}$ & $28 \pm 8$ & $22.2 \pm 5.6$ & $13.4 \pm 2 * *$ \\
\hline PA & $4.5 \pm 0.8$ & $4.0 \pm 0.7$ & $3.54 \pm 1.51 * * *$ & $4.4 \pm 1.2$ & $4.13 \pm 0.6$ & $2.4 \pm 0.2 *$ \\
\hline $\mathrm{BMC}$ & $23.7 \pm 5.4$ & $23.5 \pm 4.9$ & $16.02 \pm 5.2 *$ & $18.8 \pm 6.6$ & $17.4 \pm 3.5$ & $10.0 \pm 2 * *$ \\
\hline AEI & $8.47 \pm 1.34$ & $7.02 \pm 1.10$ & $6.98 \pm 1.48 * * *$ & $8.9 \pm 1.7$ & $7.76 \pm 1.4$ & $4.9 \pm 0.2$ \\
\hline BMI-c & $6.49 \pm 1.41$ & $6.41 \pm 1.07$ & $6.33 \pm 1.89$ & $6.96 \pm 2.55$ & $6.49 \pm 1.22$ & $13.7 \pm 0.5^{* *}$ \\
\hline
\end{tabular}

$* \mathrm{p}<0.05$

$* * \mathrm{p}<0.01$

$* * * \mathrm{p}<0.05$ vs CRF 


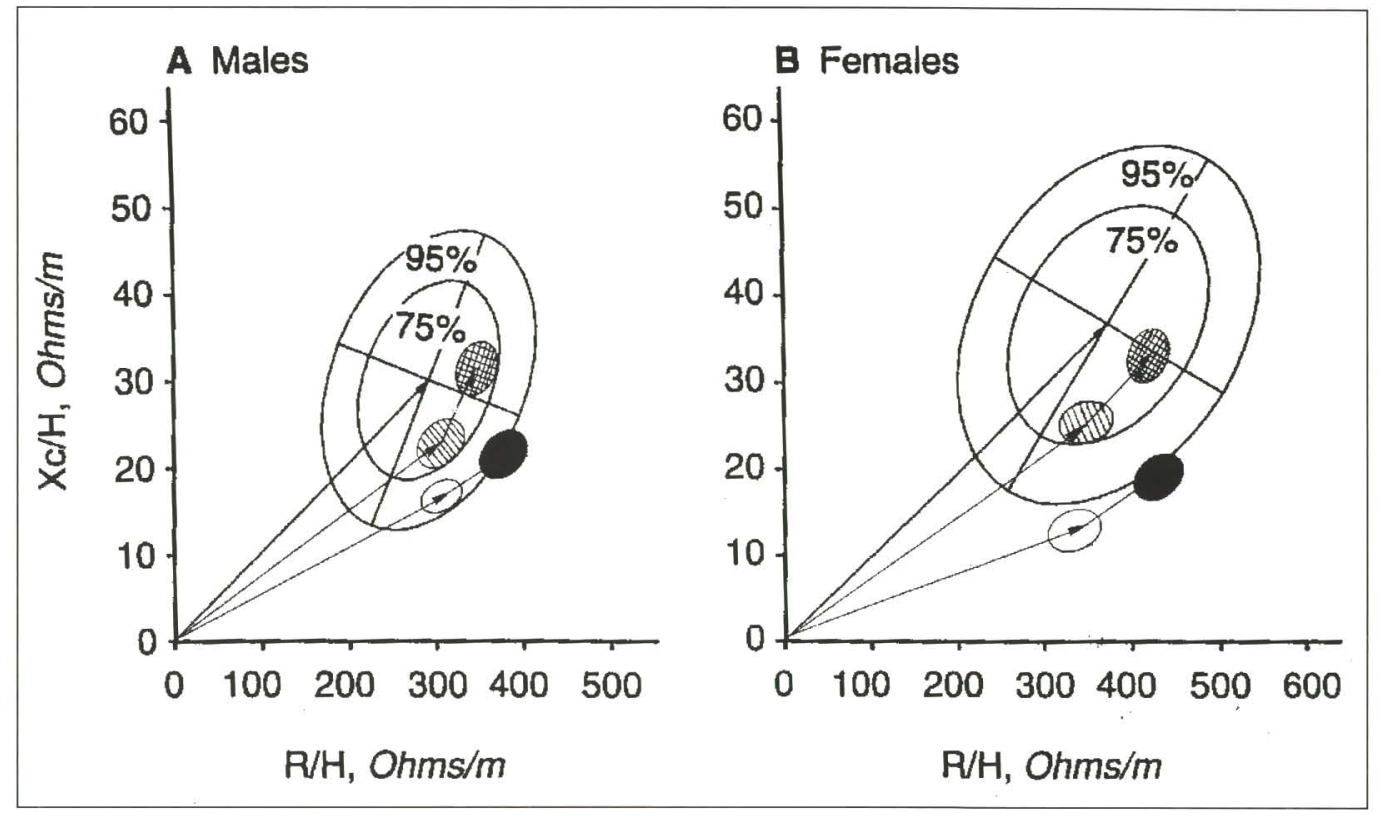

Fig. 1 - RXc-graph in pazienti emodializzati distinti per sesso. La freccia indica $i$ vettori dei pazienti vivi (ellisse a righe) e morti (ellisse vuota) in pre-dialisi. In post-dialisi, la freccia piccola indica lo spostamento del vettore, nei vivi (ellisse a quadri) e nei morti (ellisse piena). Piccoli et al: The RXc graph.

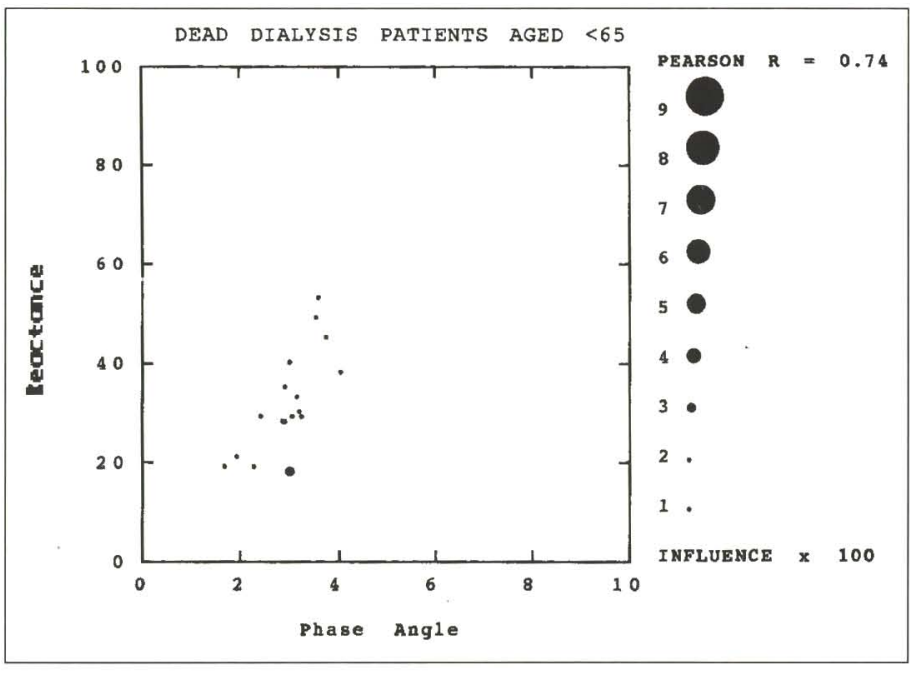

Fig. 2 - Misurazioni BIA di pazienti deceduti (con età anagrafica inferiore a 65 anni) e valutazione mediante Biagramma di Talluri.

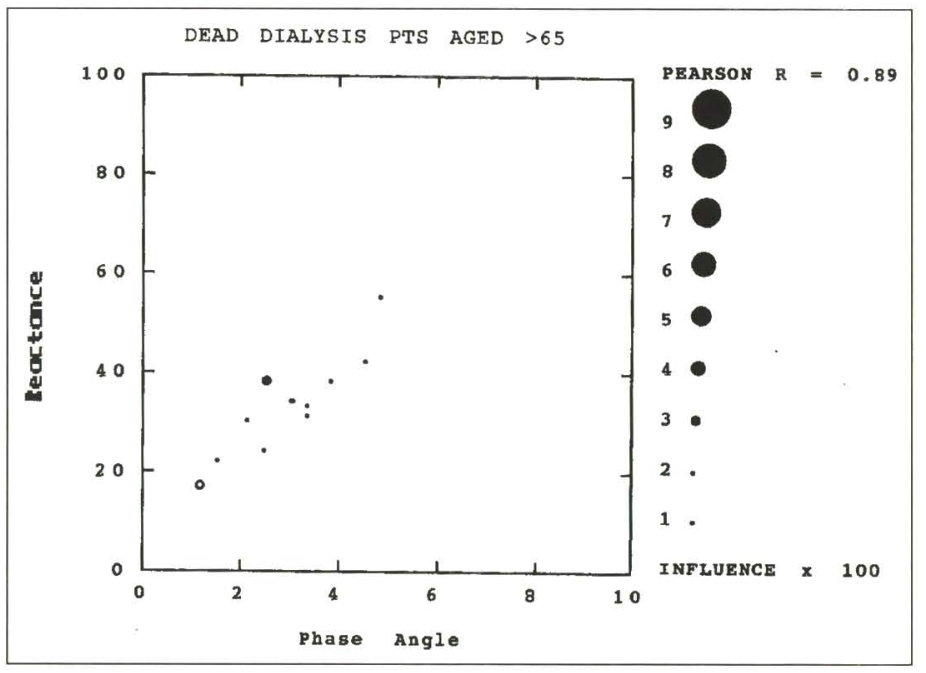

Fig. 3 - Misurazioni BIA di pazienti deceduti (con età anagrafica superiore a 65 anni) e valutazione mediante Biagramma di Talluri. $(\mathrm{r}=0.92)$ tra BCM stimata mediante BIA e quella determinata mediante metodiche di riferimento (DEXA e spazio di distribuzione di $\mathrm{NaBr}$ ). Kim et al (23) hanno mostrato come $\mathrm{Xc} / \mathrm{Re}$ sembri essere associato, più di altri classici indici nutrizionali, con FFM e quindi possa essere utilizzato come marker di malnutrizione. Ancora, Maggiore et al (24) hanno trovato correlazioni significative tra PA e vari indici nutrizionali chimico-clinici (albumina sierica, SGA, età, nPCR e misure antropometriche).

Infine, anche V. Joyeux, M.P. Morin et al (25) giungono alle stesse conclusioni di Maggiore.
Esistono, però, ancora molti dubbi circa l'affidabilità dei parametri BIA come indici nutrizionali specifici. Comunque, soprattutto PA, se considerato come indice generico, ha implicazioni cliniche sovrapponibili a quelle dell'albumina sierica: ciascuno dei due tende a riflettere la severità dello stato di malnutrizione e, pertanto, entrambi spesso appaiono alterati proprio nei pazienti più compromessi (23).

L'importanza data all'assetto nutrizionale in HD nasce dall'osservazione che molti indici in merito sono significativamente associati con la sopravvivenza del paziente. Biasioli (26) ha elaborato, con la misura della capacità elettrica, un nomogramma per la valutazione dell' "Indice di Benessere". Questo Autore, infatti, ha trovato, in una popolazione di 56 emodializzati, che la Capacità Elettrica in pre-dialisi presentava un range tra 537.000 e 1.821 .000 picofaraday e che nella stessa popolazione il $58.3 \%$ dei pazienti con Capacità $<750.000$ decedeva entro 6 mesi dalla misurazione, mentre nella popolazione con Capacità > 750.000 solo il $2.27 \%$ dei pazienti era deceduto nello stesso periodo.

Chertow et al (27), in una popolazione di ben 3.009 emodializzati, durante un follow-up a 12 mesi, hanno trovato che 


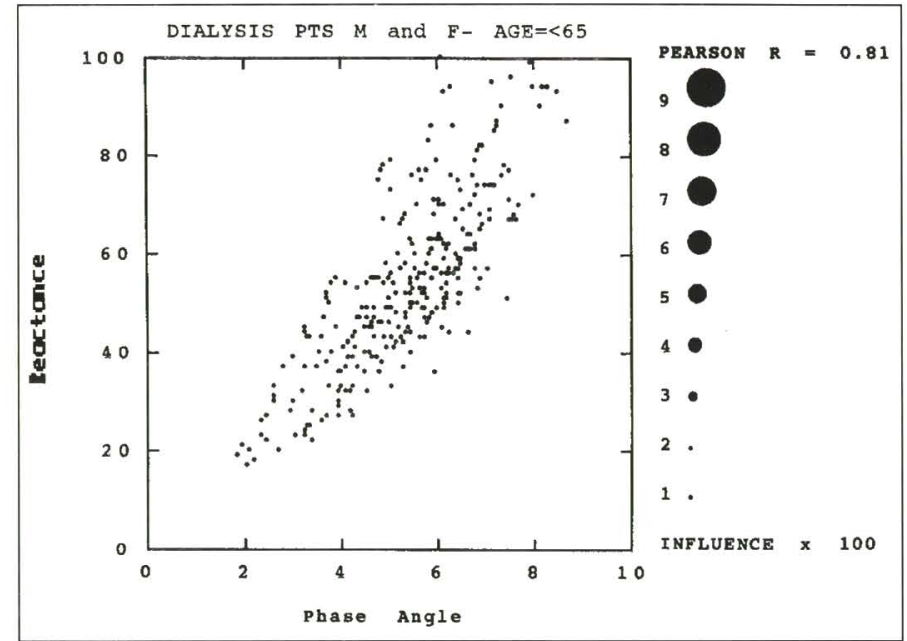

Fig. 4 - Misurazioni BIA di pazienti vivi (con età anagrafica inferiore a 65 anni) e valutazione mediante Biagramma di Talluri.

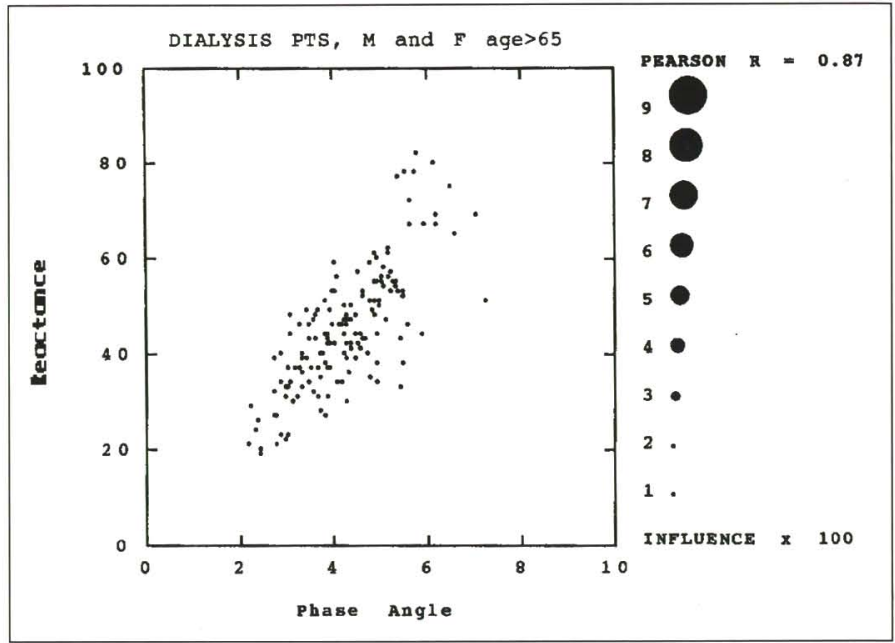

Fig. 5 - Misurazioni BIA di pazienti vivi (con età anagrafica superiore a 65 anni) e valutazione mediante Biagramma di Talluri.

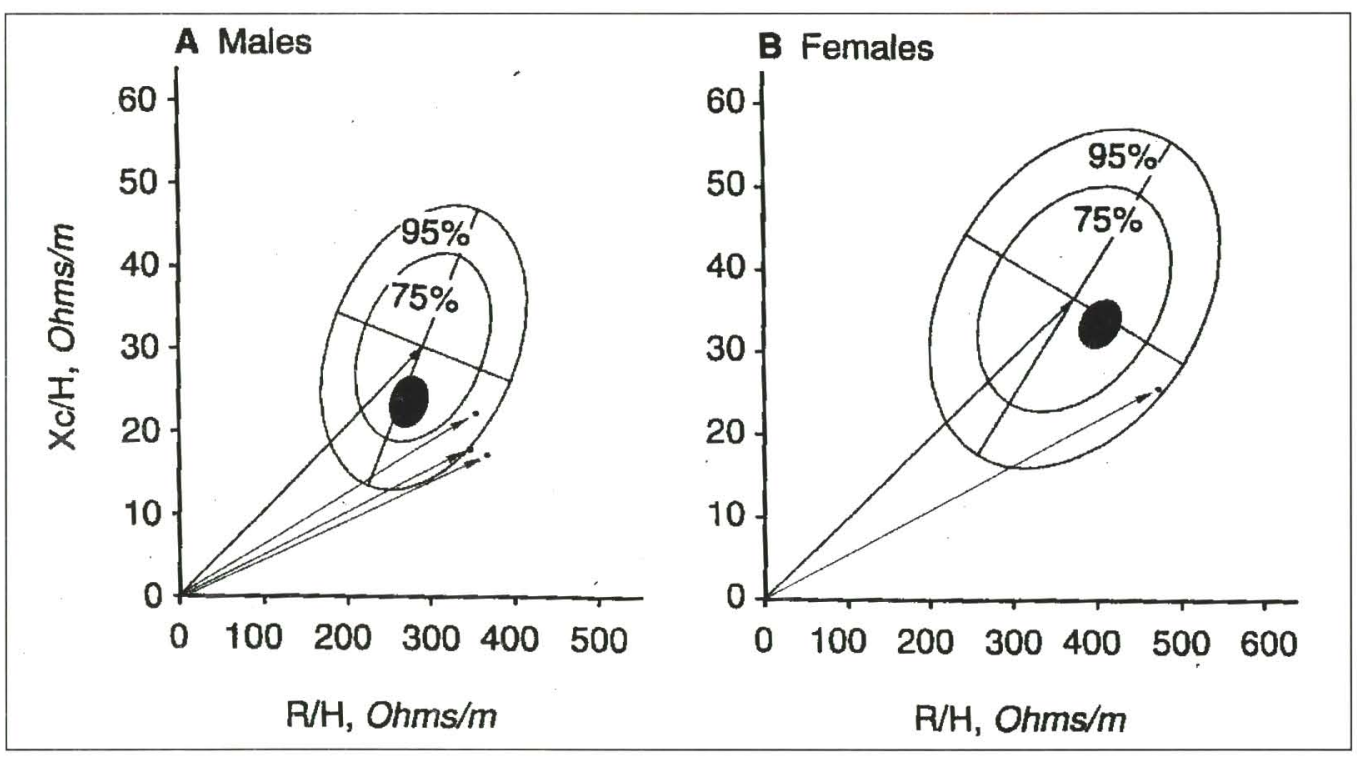

Fig. 6 - $R X c$ graph in pazienti anziani con IRC $e$ in pazienti deceduti di entrambi $i$ sessi. Le frecce con il punto indicano le misure dei pazienti deceduti. Piccoli et al: The RXc graph.

$\mathrm{Xc} / \mathrm{Re}$ ha una valenza come indice prognostico di sopravvivenza.

Ancora, Maggiore et al (24) individuano PA come efficace fattore prognostico di mortalità in HD.

Anche il nostro gruppo ha affrontato il problema della possibilità di usare i dati BIA come predizione della sopravvivenza sia in HD che in pazienti anziani con IRC.

Abbiamo studiato una popolazione non selezionata di 51 pazienti $(30 \mathrm{M}, 21 \mathrm{~F})$ in HD presso il nostro centro con un follow-up di 4 anni. Durante il periodo di osservazione sono deceduti 6 pazienti. La valutazione delle misure elettriche ha dimostrato che Xc post-HD, PA, BCM, BMI-c (Body Mass Index/PA), AEI (Indice di Adeguatezza Elettrica) (27) ed età anagrafica mostrano differenze statisticamente significative $(\mathrm{p}<0.001)$ tra $\mathrm{i}$ pazienti ancora vivi e quelli deceduti $(28,29)$. Inoltre, l'età dialitica era significativamente più bassa $(\mathrm{p}<0.001)$ nei morti rispetto ai pazienti vivi (29). Xc pre-HD e albumina mostravano differenze significative $(\mathrm{p}<0.05)$ nelle $\mathrm{F}$, ma non nei M; la Capacità Elettrica mostrava differenza significativa nei $\mathrm{M}(\mathrm{p}<$ 0.05), ma non nelle F (Tabb. I-II) (29).

Infine, la valutazione mediante RXc graph (30) ha mostrato che i pazienti morti, in entrambi i sessi, mostravano un vettore che si posizionava al di fuori (con un PA ridotto) dell'ellissi di tolleranza al $75 \%$ (Fig. 1).

Anche la valutazione mediante Biagramma di Talluri (14) mostra che le misure elettriche possono rappresentare un valido fattore predittivo della mortalità. Infatti dopo 4 anni di osservazione 14/17 $(82.35 \%)$ determinazioni BIA post-dialitiche di pazienti con età inferiore a 65 anni e 10/12 di pazienti (83.33\%) con età superiore a 65 anni deceduti avevano mostrato valori di iperidratazione (Figg. 2-3); invece nei soggetti ancora vivi le determinazioni BIA mostravano valori di iperidratazione in solo il $20 \%$ dei pazienti con età inferiore a 65 anni (Fig. 4) e nel $35 \%$ di quelli con età superiore a 65 anni (Fig. 5).

Ancora, in pazienti anziani ( $\mathrm{n}=40$ pazienti, età anagrafica $>75$ anni) con IRC, abbiamo dimostrato (31-33) che Re, Xc, albumina, acido urico, creatinina, trigliceridi e colesterolo, bicarbonati e, infine, 
emoglobina non differivano tra i pazienti ancora vivi (follow-up di 30 mesi) e i 4 pazienti (3 M, $1 \mathrm{~F}$ ) deceduti. Invece, PA mostra differenze significative $(p<0.05)$ tra $\mathrm{i}$ due gruppi, in entrambi i sessi, così come BCM e AEI $(\mathrm{p}<0.05$ in $\mathrm{M}$; $\mathrm{p}<$ 0.01 in F) (Tab. III).

Ancora, la valutazione con RXc graph mostra che la linea di confine rappresentata dall'ellissi di tolleranza al $75 \%$ (con riduzione di PA) può essere definita quale importante fattore predittivo di mortalità in questo gruppo di pazienti (Fig. 6) (31-33)

\section{Conclusioni}

La necessità di poter disporre di dati della composizione corporea che siano affidabili e riproducibili è un'esigenza indispensabile nella pratica medica pluridisciplinare.

La BIA consente facilmente di poter disporre di tali dati della composizione corporea e dello stato nutrizionale, della quantificazione dell'acqua totale e della sua compartimentazione.

Appare dimostrato come le misure BIA siano utilizzabili quali indici nutrizionali. Infine, esistono sempre più evidenze che le misure elettriche possono essere favorevolmente utilizzate quali predittrici di mortalità nei pazienti con IRC e in quelli in dialisi, come già dimostrato per i pazienti con infezione HIV (34).

Certamente, nuovi studi e l'uso di bioimpedenziometri di recente generazione possono migliorare la comprensione dell'uso della BIA e meglio determinare le sue implicazioni in clinica $(35,36)$.

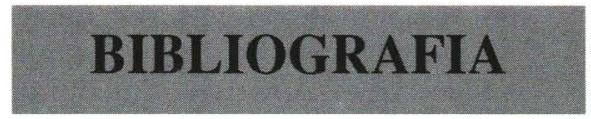

1. Kushner RF, Schoeller DA, Field CR, Danford L. Is the impedance index (ht $2 / \mathrm{r})$ significant in predicting TBW? Am J Clin Nutr 1992; 56: 835-9.

2. Lukaski HC. Methods for the assessment of human body composition: traditional and new. Am J Clin Nutr 1987; 46: 537-56.

3. Kong $\mathrm{CH}$, Thompson $\mathrm{CM}$, Lewis CA, et al. Determination of total body water in uraemic patients by bioelectrical impedance. Nephrol Dial Transplant 1993; 8: 716-9.

4. Nigrelli S, Pizzarelli F. Bioelectrical impedance analysis: a non-invasive assessment of body composition in uremics. J Nephrol 1989; 2: 155-6.

5. Biasioli S, Talluri T. La bioimpedenza in nefrologia. Egida, Vicenza 1995.

6. Segal KB, Burastero $\mathrm{S}$, Chun A, et al. Estimation of ECW and TBW by multiplefrequency bioelectrical impedance measurement. Am J Clin Nutr 1991; 54: 26-9.

7. Segal KB, Butin B, Presta E, et al. Estimation of human body composition by electrical impedance methods: a comparative study. J Appl Physiol 1985; 58: 1565-71.

8. Shizgal HM. Determination of body composition from bioelectrical impedance. Am J Parent and Enter Nutrition 1989; 4: 4557.

9. Ho LT, Kushner RF, Schoeller DA, et al. Bioimpedance analysis of TBW in hemodialysis patients. Kidney Int 1994; 46: 1438-43.

10. Di Iorio B, Talluri T, Terracciano V, Altieri C. Valutazione della composizione corporea mediante indici derivati dalla bioim- pedenziometria. Gior Ital Nefrol 1996; 13: 105-11.

11. Piccoli A, Rossi B, Pillon L, Bucciante G. A new method for monitoring body fluid variation by bioimpedance analysis: the RXc graph. Kidney Int 1994; 46: 534-9.

12. Piccoli A, Nigrelli S, Caberlotto A, et al. Bivariate normal values of the bioelectrical impedance vector in adult and elderly populations. Clin Nutr 1995; 61: 269-70.

13. Piccoli A, Rossi B, Pillon L. Is $50 \mathrm{KHz}$ the optimal frequency in routine estimation of body water by bioelectrical impedance analysis? Am J Clin Nutr 1992; 56: 1069 .

14. Piccoli A, Rossi B, Pillon L. Operational equivalence between segmental and whole-body bioelectrical impedance in renal patients. The Amer J Clin Nutrit 1994; 59 (S3): 675-6.

15. Talluri A, Maggia G. Bioimpedance analysis in hemodialysis: technical aspects. The Int $\mathbf{J}$ of Artif Organs 1995; 18: 687-92.

16. Biasioli S, Foroni F, Scudelotti F, et al. Parametri bioelettrici degli emodializzati. Analisi Statistica con le polinomiali di $3^{\circ}$ ordine e con le interpolate logaritmiche. Nefrologia veneta 1996 ; 16: 4-21.

17. De Palo T, Mariani V, Messina $\mathrm{G}$, et al. La Bioimpedenziometria nel bambino. Standard di riferimento in una popolazione prepubere del sud Italia. Atti del XIII Convegno Interregionale di Nefrologia, Sez. Apulo-Lucana, Putignano 20-21 ottobre 1995; 385-97.

18. De Palo T, Mariani V, Caringella $\mathrm{AD}$, Dammacco F. Valutazione mediante BIA della composizione corporea in bambini prepuberi del sud Italia. Gior Ital Nefrol 1996; 13: 397-404.

19. Bazzato G, Fracasso A, Morachiello $\mathrm{P}$, et al. Bioimpedance 
to assess adequate dialysis. Blood purification in perspective: new insights and future trends, vol. II, Man NK, Botella J, Zucchelli P, eds. ICAOT press, Cleveland 1992; 319-23.

20. Chertow GM, Lowrie EG, Wilmore DW, et al. Nutritional assessment with Bioelectrical Impedance analysis in maintenance hemodialysis patients. J Am Soc Nephrol 1995; 6: 75-81.

21. Nentchev N, Kozlovski P, Todorov K, Marinova E. Bioimpedance technique for determination of the adipose tissue in diabetic dialysis patients. Nephrol Dial Transplant 1996; 11: A121.

22. Inigo PJ, Lou LM, Sanz A, et al. Bioimpedance index to evaluate the nutritional status in hemodialysis patients. Nephrol Dial Transplant 1996; 11: A219.

23. Kim MJ, Hong SB, Lee SW $\mathrm{Xc} / \mathrm{R}$ ratio derived from Bioelectrical Impedance Analysis as a nutritional marker in hemodialysis patients. Abstracts of the 1996 Annual Meeting of American Society of Nephrology, 3-7. November 1996.

24. Maggiore Q, Nigrelli S, Ciccarelli $\mathrm{C}$, et al. Nutritional and prognostic correlates of bioimpedance indexes in hemodialysis patients. Kidney Int 1996; 50: 2103-8.

25. Joyeux V, Morin MP, Djeffal R, et al. SGA, Urea kinetic, Body composition analysis by multifrequency bioimpedance in hemodialyzed patients. Abstracts of the 1996 Annual Meeting of American Society of Nephrology, 3-7. November, 1996.

26. Biasioli S, Petrosino L, Cavallini $\mathrm{L}$, et al. La Bioimpedenza in continuo durante il trattamento dialitico: 1'indice di Benessere. Abstract XXXV Congresso Nazionale S.I.N., Bari 18-21 May 1994; 58.

27. Chertow GM, Lowrie EG, Lew NL, Lazarus JM. Bioelectrical impedance analysis predicts survival in Hemodialysis patients. Abstracts of the 1996 Annual Meeting of American Society of Nephrology, 3-7. November 1996.

28. Di Iorio B, Talluri T, Altieri $\mathrm{C}$, et al. Validazione degli indici bioimpedenziometrici-derivati. Giorn Ital Nefrol 1995; 12: 281-2.

29. Di Iorio B, Terracciano V, Altieri C, Lopez T. Mortalità e dialisi: studio prospettico in una popolazione non selezionata. Follow-up di 4 anni. Sixth Assisi European Meeting on Cardionephro$\log y$, M. Timio and V. Wizermann eds, Assisi, 10-12. April 1997; in press.

30. Di Iorio B, Terracciano V, Altieri C, Lopez T. RXc graph e mortalità in dialisi: dati preliminari in una popolazione non selezionata con osservazione prospettica di 4 anni. Giorn Ital Nefrol submitted.

31. Di Iorio B, Terracciano V, Altieri C, Lopez T. Dati BIA e mortalità in pazienti anziani con IRC. Sixth Assisi European Meeting on Cardionephrology, M. TImio and V. Wizemann eds, Assisi, 10-12. April 1997, in press.

32. Di Iorio B, Terracciano V, Lopez T. IRC e mortalità in pazienti anziani: influenza di fattori antropometrici, biochimici e bioelettrici. Gior Ital Nefrol submitted.

33. Di Iorio B, Terracciano V, Lopez T. CRF and mortality: analysis of bioelectrical, anthropometric and biochemical factors in elderly patients. $\mathrm{J}$ of Renal Nutrit submitted.

34. Ott M, Fischer H, Polat H, et al. Bioelectrical impedance analysis as a predictor of survival in patients with HIV infection. $J$ of Acquired Immune Deficiency Syndromes and human retrovirus 1995; 9: 20-4.

35. De Lorenzo A, Candeloro N, Bertini J, Talluri T. Total body capacity correlated with Basal Metabolic Rate. International symposium on Body Composition Studies, 18-20. September, Malmoe, Sweden, 63.

36. Talluri T. Assessment of Body Cell Mass and Extracellular Water without stature height and weight: a new Bioimpedance method. International Symposium on Body Composition Studies, 18-20. September, Malmoe, Sweden, 12. 